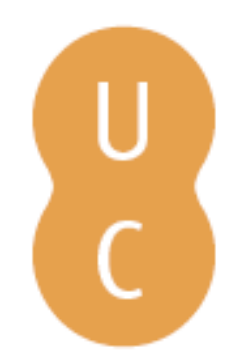

\title{
nommalina
}

\section{Sistema de informação e apoio à decisão para projetos de regeneração urbana baseado em tecnologia SIG}

Autor(es): $\quad$ Jesus, Eduardo Natividade; Rodrigues, João Coutinho; Sousa, Nuno

Publicado por: Imprensa da Universidade de Coimbra

URL

persistente: URI:http://hdl.handle.net/10316.2/37100

DOI: $\quad$ DOI:http://dx.doi.org/10.14195/978-989-26-0983-6_47

Accessed : $\quad$ 26-Apr-2023 10:47:27

A navegação consulta e descarregamento dos títulos inseridos nas Bibliotecas Digitais UC Digitalis, UC Pombalina e UC Impactum, pressupõem a aceitação plena e sem reservas dos Termos e Condições de Uso destas Bibliotecas Digitais, disponíveis em https://digitalis.uc.pt/pt-pt/termos.

Conforme exposto nos referidos Termos e Condições de Uso, o descarregamento de títulos de acesso restrito requer uma licença válida de autorização devendo o utilizador aceder ao(s) documento(s) a partir de um endereço de IP da instituição detentora da supramencionada licença.

Ao utilizador é apenas permitido o descarregamento para uso pessoal, pelo que o emprego do(s) título(s) descarregado(s) para outro fim, designadamente comercial, carece de autorização do respetivo autor ou editor da obra.

Na medida em que todas as obras da UC Digitalis se encontram protegidas pelo Código do Direito de Autor e Direitos Conexos e demais legislação aplicável, toda a cópia, parcial ou total, deste documento, nos casos em que é legalmente admitida, deverá conter ou fazer-se acompanhar por este aviso.

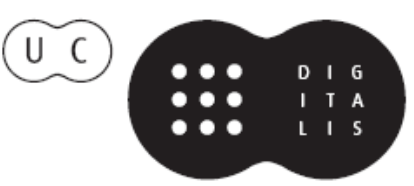




\section{$\forall$ \\ TAS DAS I JORNADAS LUSÓFONAS DE CIÊNCIAS E TECNOLOGIAS DE INFORMAÇÃO GEOGRÁFICA}

Editores

José Gomes dos Santos

Cidália Fonte

Rui Ferreira de Figueiredo

Alberto Cardoso

Gil Gonçalves

José Paulo Almeida

Sara Baptista 


\section{A RTIGO 47}

\section{SISTEMA DE INFORMAÇÃO E APOIO À DECISÃo PARA PROJETOS DE REGENERAÇÃO URBANA BASEADO EM TECNOLOGIA SIG}

NATIVIDADE-JESUS, Eduardo ${ }^{1,2}$; COUTINHO-RODRIGUES, João1,3 \& SOUSA, Nuno ${ }^{1,4}$

\footnotetext{
${ }^{1}$ Instituto de Engenharia de Sistemas e Computadores (INESC-Coimbra); Rua Antero Quental 199, 3000033 Coimbra, Portugal; Tel: +351 239 851040/9; Fax: +351 239 824692;

2 Departamento de Engenharia Civil do Instituto Superior de Engenharia de Coimbra Instituto Superior de Engenharia de Coimbra; Rua Pedro Nunes - Quinta da Nora, 3030-199 Coimbra, Portugal; Tel: +351 239 790200; Fax: +351239790311; email: ednativi@dec.uc.pt

3 Departamento de Engenharia Civil da Faculdade de Ciências e Tecnologia da Universidade de Coimbra Instituto de Engenharia de Sistemas e Computadores (INESC-Coimbra) Departamento de Engenharia Civil - FCTUC; Rua Luís Reis Santos - Pólo II, 3030-788 Coimbra, Portugal; Tel: +351 239 797145; Fax: +351239 797123; email: coutinho@dec.uc.pt

${ }^{4}$ Departamento de Ciências e Tecnologia da Universidade Aberta Instituto de Engenharia de Sistemas e Computadores (INESC-Coimbra) Delegação de Coimbra da Universidade Aberta; Rua Alexandre Herculano, n. ${ }^{\circ}$ 52, 3000-019 Coimbra, Portugal; Tel: +351 300 001590; Fax: +351 300 001599; email: nsousa@uab.pt
}

\section{RESUMO}

A Regeneração Urbana (RU) é um problema atual e importante, fundamental para a gestão das cidades, proteção do ambiente e melhoria das condições de vida. Deixou de ser apenas uma área de pesquisa para se tornar parte integrante das novas políticas urbanas e de habitação. A Regeneração Urbana diz respeito não só à reabilitação de múltiplos edifícios, mas também à requalificação das infraestruturas urbanas e de serviços. A regeneração urbana envolve assim decisões complexas, fruto das múltiplas dimensões do problema que incluem, entre outros, critérios técnicos de engenharia, preocupações com a segurança, custos financeiros, socioeconómicos e ambientais, etc. A natureza multidimensional da 
regeneração urbana e a dimensão dos investimentos que lhe estão associados justificam o desenvolvimento e utilização das mais recentes tecnologias e metodologias de apoio à decisão, de modo a apoiar e ajudar os agentes de decisão (AD) na obtenção de melhores e mais sustentadas soluções. Apresenta-se um Sistema Web de Apoio à Decisão Espacial (web-SADE), desenvolvido para auxiliar os AD na análise do problema e na definição de estratégias e prioridades de intervenção de RU. Integra um módulo de gestão de base de dados, um Sistema de Informação Geográfica (SIG), uma base de métodos de análise multicritério, e um interface humano-computador adequado. É ainda apresentado um exemplo da aplicação do web-SADE, num contexto real (Projeto de Renovação Urbana da Baixa de Coimbra).

\title{
PALAVRAS-CHAVE
}

Análise multi-critério, Sistemas de apoio à decisão espaciais, Regeneração urbana, Web-SIG.

\section{GIS BASED INFORMATION AND DECISION SUPPORT SYSTEM FOR URBAN REGENERATION PROJECTS}

\begin{abstract}
Urban Regeneration is an actual and important problem, of fundamental importance for the management of cities, environmental protection, and general improvement of life conditions. It is no longer only an area of study and research; it became part of the new urban and housing politics. It addresses not only the renovation of large numbers of buildings but also the redesign of the urban infrastructures and services. Such efforts involve complex decisions as a consequence of the multiple dimensions of the problem that include special technical engineering requirements, safety concerns, financial costs and socio-economic, environmental, aesthetic, political impacts, among others. This multidimensional nature of urban regeneration projects and their large capital investments justify the development and use of state-of-the-art decision support methodologies to assist Decision Makers (DM). This research introduces a web Spatial Decision Support System (web-SDSS), developed for aiding the DM in the analysis of the problem and in the definition of strategies and priorities of intervention. It integrates a data base management module, a Geographic Information System (GIS), a Multicriteria analysis methods base, and an adequate Human-computer interface - aimed at minimizing the cognitive effort required from users. An example of the application of the web-SDSS to a real-world context (the Renovation Project of Coimbra Downtown) is presented.
\end{abstract}

\section{KEYWORDS}

Multiple criteria analysis, Spatial decision support systems, Urban regeneration, Web-GIS. 


\section{INTRODUÇÃO}

\subsection{O contexto}

O consumo de energia e aquecimento global são duas das maiores preocupações económicas e ambientais mundiais, como o demonstra o Protocolo de Kyoto (UNFCCC 1997), ratificado pela União Europeia (UE) em 31 de maio de 2002. Este protocolo refere que o planeamento urbano pode ter um papel importante no esforço para reduzir o consumo de energia, o aquecimento global e assim contribuir para um desenvolvimento sustentável. O protocolo apela a uma adaptação de "[...] tecnologias e métodos para melhoramento do planeamento espacial" para atingir os seus objetivos no que toca à eficiência energética e redução de poluição.

Tal como indicam o Protocolo de Kyoto e a Diretiva 2002/91/CE do Parlamento Europeu e do Conselho, relativa ao desempenho energético dos edifícios (UNIÃO EUROPEIA, 2003), recentemente reformulada pela Diretiva 2010/31/CE, de 19 de Maio (UNIÃO EUROPEIA, 2010) a renovação de edifícios existentes (e áreas dos centros urbanos) é um fator crucial para um uso mais eficiente de energia (DING, 2008, CAPUTO et al., 2012).

De facto, o sector dos edifícios representa cerca de 39\% do consumo mundial de energia primária e 34\% das emissões de dióxido de carbono (CO2) (PRICE et al., 2006), não sendo por isso de estranhar que o diretor executivo da Agência Internacional de Energia- AIE, Nobuo Tanaka, afirme que "[...] para um futuro energético sustentável, o sector dos edifícios deve desempenhar um papel fundamental”.

Como se pode observar da análise da Figura 1, na U.E. o sector dos edifícios representava em 2010 41\% do consumo final de energia, sendo assim o sector com maior peso, à frente do sector dos transportes (com 32\%) e do da indústria com (25\%).

Assim, tendo em conta o enorme estoque de edifícios existentes, a sua longevidade e a generalizada ineficiência energética, é na sua reabilitação que a longo prazo reside o maior potencial de redu- 
ção do consumo de energia (ZAVADSKAS et al., 2008, COPENHAGEN ECONOMICS, 2012).

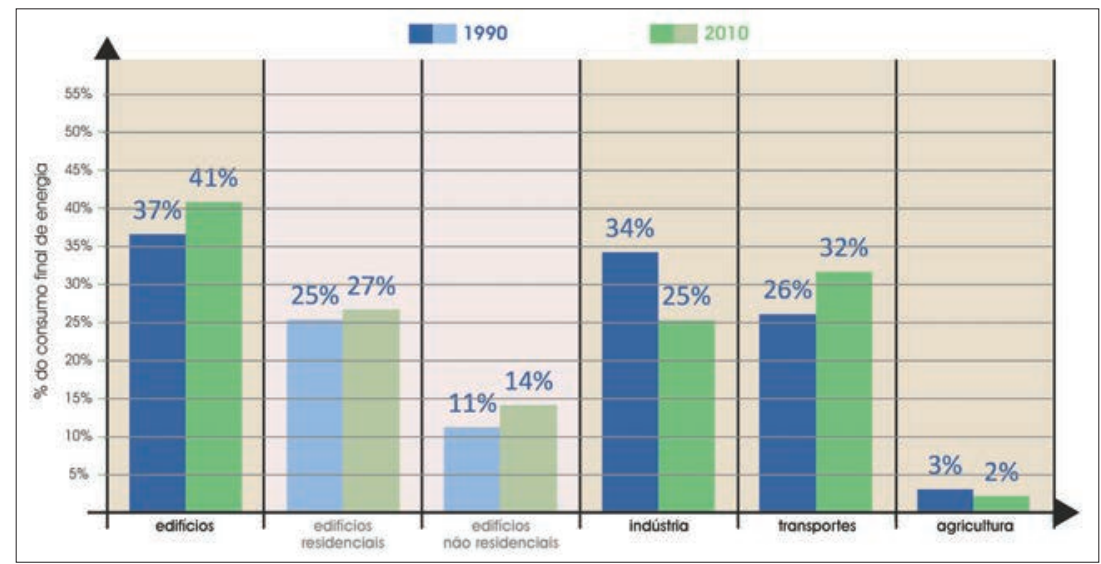

Figura 1 - Consumo final de energia - U.E. por sectores (adaptado de ENERDATA \& ADEME, 2012)

Por comparação com outros países europeus Portugal está atrasado na aplicação do Protocolo de Kyoto ao desenvolvimento urbano. De facto, Portugal tem atualmente 5859540 habitações (CENSOS, 2011), das quais 3062835 foram construídas depois de 1981 (52\% do total habitações). Significa isto que nos últimos 30 anos se construíram em Portugal uma média de 100000 habitações por ano. Assim, o número atual de habitações é $45 \%$ superior ao número de famílias (mais 1,8 milhões de casas do que famílias), o que faz com que Portugal tenha uma das maiores taxas de habitações por família da Europa - 1.4 habitações por agregado familiar. No entanto, uma parte significativa do parque habitacional está envelhecido e/ou degradado - estima-se que 29\% dos edifícios residenciais existentes (1 024937 de edifícios) necessitam de obras de reabilitação e esta percentagem sobe para $43 \%$ nos edifícios com mais de 30 anos. Destes últimos, 19\% necessitam de pelo menos reparações médias e $8 \%$ necessitam de grandes reparações ou estão muito degradados.

Apesar deste cenário a regeneração urbana, e em particular a reabilitação de edifícios existentes, tem em Portugal uma expressão ainda relativamente reduzida face às necessidades reais e quando comparada 
com o que acontece na grande maioria dos países da União Europeia.

De facto, o segmento de renovação e manutenção ( $R$ \& $M$ ) de edifícios residenciais representa apenas cerca de $23 \%$ do volume total da construção de edifícios em Portugal (fonte: INE, 2010), enquanto que em outros países EuroConstruct ${ }^{1}$ o sector da R \& M de edifícios de habitação já representa aproximadamente $60 \%$ do mercado residencial total (como por exemplo, no Reino Unido, na Bélgica, na Noruega, na Holanda, na Alemanha, na Dinamarca, na França e na Itália) (EUROCONSTRUCT, 2009).

No entanto, nos últimos anos a regeneração urbana tornou-se um tema cada vez mais frequente na agenda pública e política portuguesa (ver BREDA-VÁZQUEZ, 2009), parecendo existir agora uma consciência cada vez maior sobre a importância e premência da regeneração urbana, em especial nas áreas centrais e históricas das nossas cidades, como o comprova uma série de legislação recente. Senão vejamos:

a) O Decreto-Lei 104/2004 (DIÁRIO DA REPÚBLICA, 2004) veio criar as Sociedades de Reabilitação Urbana, com o intuito de alargar o âmbito das ações de reabilitação das habitações individuais para uma escala de bairro. Estas sociedades têm atribuições e poderes especiais no que toca à expropriação e licenciamento, tornando possível uma intervenção mais eficiente.

b) O documento das Grandes Opções do Plano para 2005-2009, Lei ${ }^{\circ}$ 52/2005 (DIÁRIO DA REPÚBLICA, 2005) designou a regeneração urbana como um dos cinco principais vetores de atuação para esse período, apontando a uma maior qualidade ambiental, melhor ordenamento e gestão, coesão territorial e melhores cidades.

c) A Estratégia Nacional para o Desenvolvimento Sustentável 20052015 (DIÁRIO DA REPÚBLICA, 2007) menciona igualmente a adoção,

1 O EUROCONSTRUCT, criado em 1975, é uma rede Europeia de 19 Institutos, de 19 países (Áustria, Bélgica, Dinamarca, Finlândia, França, Alemanha, Irlanda, Itália, Holanda, Noruega, Portugal, Espanha, Suécia, Suíça, Reino Unido, República Checa, Hungria, Polónia e República Eslovaca), vocacionados para a análise do sector da Construção. Organiza duas conferências por ano nas quais apresenta a evolução recente e perspectivas de evolução da economia europeia e do sector da Construção na Europa, incluindo os seus segmentos (residencial, não residencial e engenharia civil). Portugal está representado através do ITIC - Instituto Técnico para a Indústria da Construção. 
até 2010, de uma estratégia para as cidades, na qual se almeja levar a cabo ações de regeneração urbana em pelo menos $80 \%$ destas.

d) $\mathrm{O}$ quadro legal regulatório do mercado de arrendamento, Leis da República Portuguesa $n^{\circ}$ s 156/2006, 157/2006, 158/2006, 159/2006, $160 / 2006$ e 161/2006, terão provavelmente um impacto significativo no mercado de reabilitação urbana no curto e médio prazo, dado que o valor das rendas subirá como consequência de ações de regeneração nos edifícios.

\subsection{Motivação e objetivos principais deste estudo}

A regeneração urbana é um problema real, atual e com uma importância cada vez maior (principalmente nos países mais desenvolvidos), revelando-se por isso como um factor crucial nas políticas e medidas de gestão das cidades em todas as suas dimensões - económica, social, cultural, ambiental, etc. (ORUETA, 2007, COPENHAGEN ECONOMICS, 2012).

Simultaneamente decisores políticos, técnicos, especialistas e a população em geral, foram percebendo que os problemas das cidades não se resolviam apenas com intervenções no património edificado e nos espaços e infraestruturas públicas (ANDERSEN \& VAN KEMPEN, 2003). Estes problemas são bem mais complexos. De facto, eles requerem a consideração de múltiplas dimensões na análise e nas decisões, são por vezes ambíguos e com grande grau de incerteza, além de envolverem na sua resolução um cada vez maior número de intervenientes VASCONCELOS et al., 2009).

Os problemas da gestão e desenvolvimento urbano, e em particular os associados à regeneração urbana, envolvem assim múltiplas dimensões uma vez que, para além dos aspectos físicos/estruturais, contemplam também os aspectos económicos, sociais e ambientais - Figura 2.

A RU é cada vez mais encarada como uma operação complexa e multifacetada, caracterizada por uma visão mais alargada da cidade, apelando ao fomento da participação ativa de todos os interessados, com a procura de uma identificação mais nítida de todos os impactos (económico-financeiros, ambientais, sociais, culturais, etc.). É acompanhada ainda 
por uma preocupação cada vez mais notória com a sustentabilidade das intervenções.

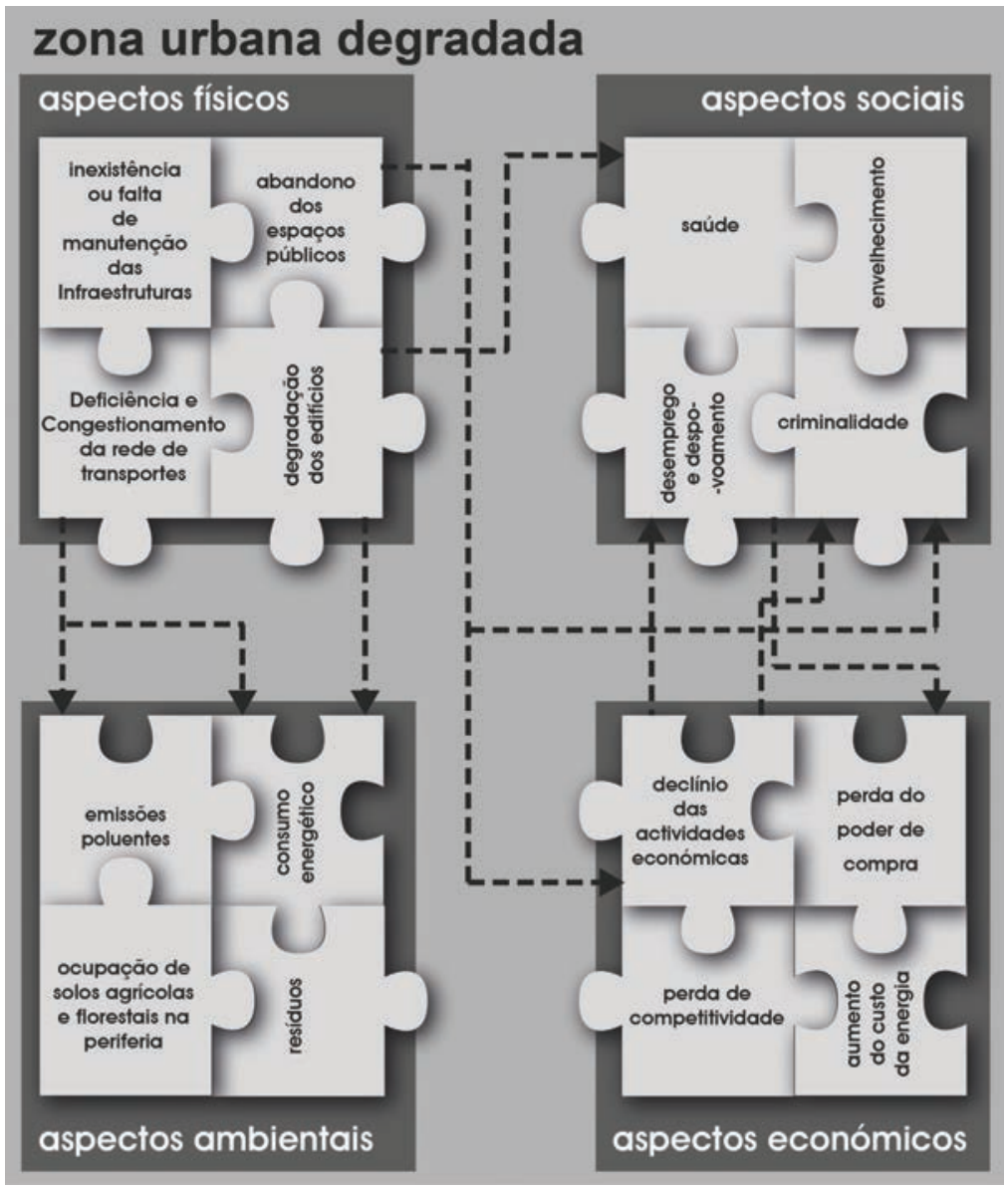

Figura 2 - Múltiplos aspectos de um processo de regeneração urbana

A RU envolve deste modo múltiplos, conflituantes e incomensuráveis aspectos na avaliação do mérito de possíveis alternativas de intervenção, dependendo do contexto do estudo e dos intervenientes (consumidores, promotores, agências governamentais ou municipais, etc.) (KAZMIERCZAK et al., 2009). As magnitudes dos benefícios e dos custos e as relações complexas e contraditórias envolvidas nestes processos vêm enfatizar a necessidade de uma abordagem integrada (BAI et al., 2010) e justificam o desenvolvimento e a utilização de metodologias avançadas de apoio aos 
agentes de decisão (NATIVIDADE-JESUS et al., 2007, et al., 2013). Estas têm vindo a ser entendidas (e partilhamos dessa opinião) como instrumentos fundamentais na estruturação e análise deste tipo de problemas, uma vez que, ao permitirem incorporar as diversas nuances e dimensões da avaliação, são mais representativas do contexto real de decisão.

Dada essa diversidade e a complexidade dos fatores que influenciam este tipo de problemas e a natureza espacial e o volume de dados envolvido, os Sistemas de Apoio à Decisão Espaciais (SADE) são uma ferramenta essencial para este tipo de estudos. Os SADE integram a eficiência e rapidez de processamento dos sistemas de informação com modelos formais que lidam com a parte de apoio à decisão.

Nesta comunicação apresentamos um web-SADE (SADE ligado à web) que pode assistir os vários atores envolvidos em ações de regeneração urbana (correspondentes a diferentes perfis de utilizador e resultados esperados) a tomar decisões mais bem fundamentadas. Este web-SADE incorpora não só tecnologia recente e cientificamente validada no apoio à decisão espacial como também expande a própria tecnologia. O web-SADE integra:

a) Um módulo de gestão de bases de dados, que incorpora informação demográfica, económica, de engenharia, arquitetónica e ambiental, entre outras.

b) Um Sistema de Informação Geográfica (SIG), dada a natureza espacial do sistema urbano subjacente.

c) Um base de métodos multicritério, consequência da natureza multidimensional dos problemas de planeamento urbano, para fornecer aos decisores e/ou projetistas uma melhor perceção dos aspetos em conflito e natureza das soluções de compromisso.

d) Tecnologia web/Internet, para lidar com a dispersão espacial dos atores.

e) Um interface humano-computador, destinado a minimizar o esforço cognitivo exigido aos utilizadores. 
Nesta secção apresentou-se a motivação e os objetivos deste estudo, bem como a panorâmica geral do problema. Na secção 2 o web-SADE para regeneração urbana é apresentado e as suas componentes e funcionalidades são descritas, com realce para as potencialidades do uso de técnicas de análise multicritério (em particular aquelas dirigidas à classificação de alternativas em categorias pré-definidas, tais como o ELECTRE TRI) na ajuda à definição de prioridades em intervenções de regeneração. A aplicação a um caso real (Projeto de Renovação Urbana da Baixa de Coimbra) é descrita na secção 3.

\section{DESENVOLVIMENTO DE UM WEB-SADE PARA INTERVENÇÕES DE REGE- NERAÇÃO URBANA}

Nos últimos anos assistiu-se a uma mudança de paradigma na natureza do planeamento urbano. Esta mudança está associada a um cada vez maior envolvimento de não-peritos nos processos de planeamento e tomada de decisões (e.g. público em geral, grupos de interesses, comunidades, organizações não-governamentais, etc.) (MALCZEWSKI, 2004).

Para além desta transformação devemos ainda compreender a dimensão temporal, espacial e até mesmo institucional das políticas urbanas, cujos impactos tendem a ultrapassar os limites da própria zona, cidade ou região, mas que, no entanto, são frequentemente esquecidos ou ignorados pelos agentes de decisão (nomeadamente os políticos). Tais impactos tendem também a ficar confinados aos interesses da sua escala temporal, aos limites espaciais da sua área de jurisdição e dentro dos seus (por vezes "limitados") contextos institucionais e organizacionais (BAI et al., 2010).

É por isso urgente uma percepção mais profunda destes impactos e consequentemente uma melhor e mais eficaz integração das políticas de sustentabilidade globais nas intervenções e práticas de gestão urbana, obrigando os intervenientes e decisores locais a transformar significativamente a sua forma "tradicional" de governar, reescalonando os seus horizontes temporais e espaciais, interagindo e coordenando ações com outros decisores locais, nacionais e "globais" (BAI et al., 2010). O fomento de uma efetiva participação pública, mais do que uma "moda", é 
uma necessidade e um caminho para a obtenção de melhores políticas públicas para potenciar o sucesso e eficácia e para reduzir eventuais conflitos (LANE, 2005).

Dada a diversidade e complexidade dos atributos, suas inter-relações, a natureza espacial e o volume de dados envolvido na regeneração urbana, a integração de tecnologias SIG com tecnologias de gestão de bases de dados e métodos multicritério afigura-se-nos como uma forma promissora de analisar projetos de regeneração urbana. Ao integrar a eficiência e a facilidade de utilização e processamento dos dados e a intuitiva representação geográfica dos dados (maioritariamente geoespaciais) com modelos formais de apoio à decisão, um SADE pode de facto ajudar os vários intervenientes a tomar decisões mais consistentes e fundamentadas nos processos de desenvolvimento urbano e em particular nos que envolvem intervenções de regeneração urbana.

A definição e prossecução destes objectivos estiveram na base do desenvolvimento do SADE cujas principais componentes são apresentadas na Figura 3.

Tendo em conta a heterogeneidade do conjunto de potenciais utilizadores e mesmo a sua eventual distribuição geográfica pareceu-nos ser mais interessante e conveniente a opção por um SADE capaz de funcionar de forma aberta e distribuído no espaço. Como tal, optou-se por desenvolver um sistema que funciona em ambiente Web - constituindo-se assim num Web-SADE.

Outras duas vantagens desta opção (ambiente Web) podem e devem ser destacadas:

a) $\mathrm{O}$ facto de qualquer potencial utilizador do sistema necessitar apenas de um computador com ligação à Internet, munido de um browser (Internet Chrome $^{\mathrm{TM}}$, Safari ${ }^{\mathrm{TM}}$, Explorer ${ }^{\mathrm{TM}}$, Firefox ${ }^{\mathrm{TM}}$, etc.);

b) O facto de permitir uma redução futura dos custos de aquisição e atualização de hardware e software, uma vez que, ao concentrar as necessidades de processamento do lado do servidor, não obrigar à aquisição (para cada um dos postos de utilização) de computadores com requisitos elevados (processador, memórias, placas gráficas, etc.) e por isso relativamente dispendiosos. 


\subsection{Arquitetura do web - SADE}

O SAD foi orientado para fornecer ajuda à decisão ao nível da avaliação do estado de conservação dos edifícios. Tal como indicado na figura 3, é composto por:

1. Uma base de dados, dotada de um sistema de gestão relacional.

2. Um servidor SIG (ArcGIS server).

3. Uma base de métodos para apoio à decisão, incluindo métodos tais como Simple Additive Weighthing (CHURCHMAN \& ACKOFF 1954, YOON \& HWANG, 1995), TOPSIS (MASSAM, 1988, YOON \& HWANG, 1995), ELECTRE I e ELECTRE TRI (ROY 1991, SCHÄRLIG, 1996).

4. Um interface web, projetado para ser user-friendly e intuitivo.

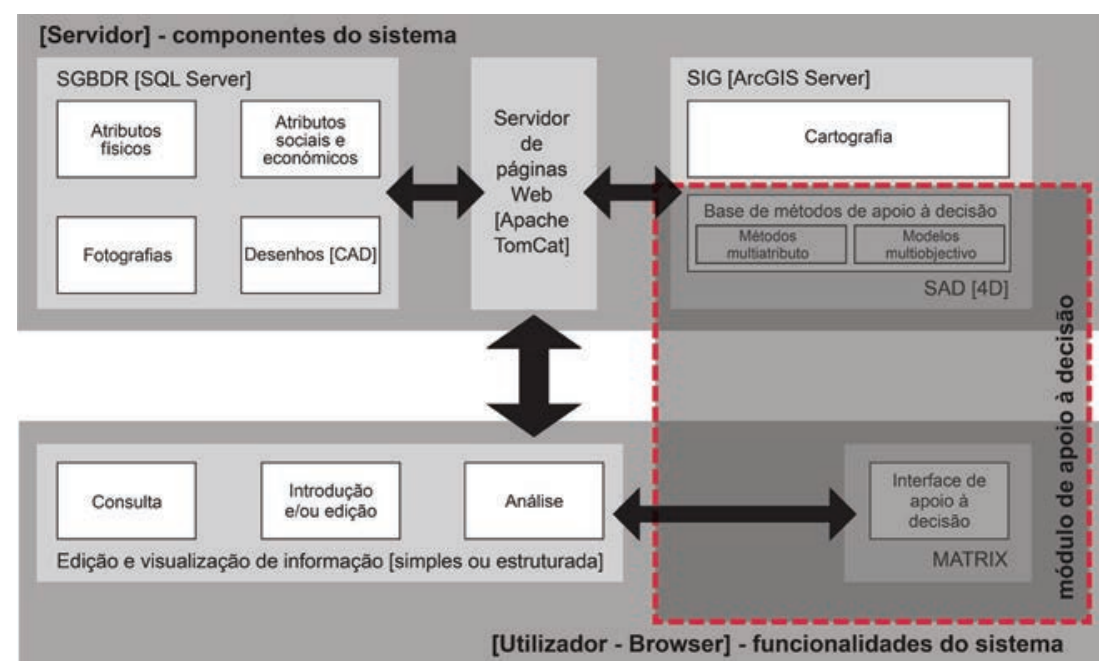

Figura 3 - Arquitetura do web-SADE

O interface permite ao utilizador não só aplicar um dos vários métodos multicritério disponíveis na base de métodos mas também visualizar, modificar, adicionar e analisar (estatisticamente) informação guardada na base de dados. O utilizador pode criar, gravar, modificar ou eliminar atributos e problemas/resultados de análise. Todas estas operações são feitas através do interface web . 


\subsection{Sistema de gestão da base de dados}

Os componentes principais do sistema de gestão da base de dados relacional (SGBDR) são:

Base de dados de atributos que caraterizam a qualidade do edifício, que inclui por exemplo atributos físicos e ambientais, e que pode ser editada pelo utilizador. Os utilizadores têm no entanto perfis de permissões diferentes, criados de forma a garantir a integridade e coerência da informação.

Base de dados de imagens de cada edifício, organizadas em oito classes: telhado, paredes, chão, escadas, cozinha e casas-de-banho, redes de água/esgotos/ eletricidade/gás, vizinhança, outras. Estas imagens podem ser vistas e/ou editadas conforme o perfil do utilizador (figura 4).

Base de dados dos projetos técnicos dos edifícios (CAD, arquitetura), disponíveis para impressão ou descarregamento.

Base de dados de problemas e resultados de análise. Esta base contém descrições de análises feitas por utilizadores, que podem servir como ponto de partida para análises subsequentes.

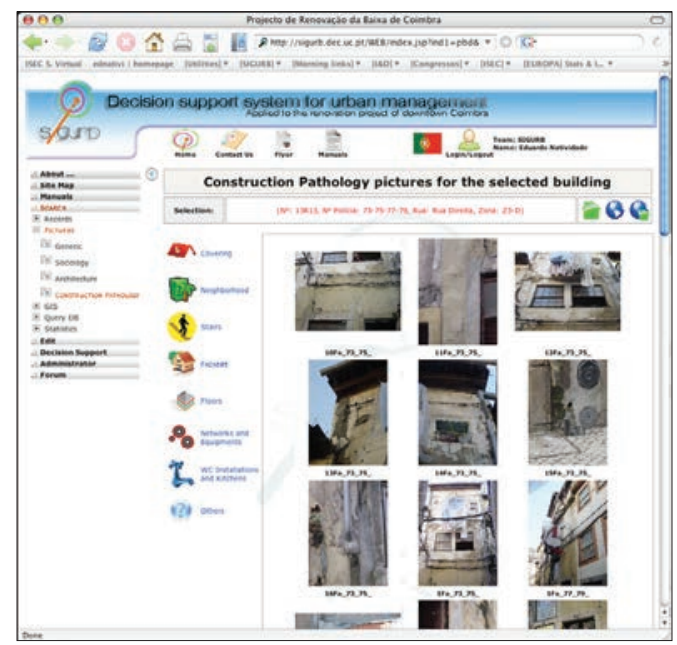

Figura 4 - Visualização da base de dados de imagens com um browser de web

\subsection{Base de métodos de apoio à decisão}

A base de métodos de apoio à decisão foi desenvolvida e progra- 
mada em 4th Dimension (4D) e 4D server (www.4d.com). O 4D inclui uma linguagem de programação que permite uma integração completa da manipulação de dados com modelos através de interfaces gráficos. Permite também comunicar com a SGBDR (SQL) e com o servidor web SIG (ArcGIS server) via controladores (drivers) ODBC.

Como mencionado acima, prestou-se especial atenção ao design e desenvolvimento deste web-SADE por forma a oferecer aos utilizadores um ambiente web flexível e simples de usar. Este é no entanto poderoso e tecnicamente robusto, capaz de disponibilizar aos utilizadores ajuda à compreensão das opções em aberto, para que estes possam fazer rever nestas as suas preferências através de análises interativas e experimentação.

Os métodos de apoio à decisão disponíveis nesta componente da base de dados cobrem uma gama de técnicas orientadas para objetivos específicos e coerentes. Estas técnicas (SAW, TOPSIS, ELECTRE) diferem entre si no que toca ao tipo de informação pedida ao decisor e ao tipo de resultado (output). Consequentemente, a escolha do método dependerá dos objetivos do decisor. Nos dois parágrafos abaixo é feita uma breve descrição do modus operandi destes métodos e seu âmbito de aplicação. Para detalhes, o leitor pode consultar a literatura indicada na secção 2.1.

Os métodos SAW e TOPSIS elaboram uma ordenação completa das várias alternativas. O SAW fá-lo atribuindo pesos aos vários critérios e executando uma soma pesada para todas as alternativas. O TOPSIS ordena as alternativas baseado na distância destas à solução "ideal" (composta pelos melhores valores em cada atributo) e ao "anti-ideal" (composta pelos piores valores em cada atributo). A ordenação bem definida das alternativas resultante destes métodos torna-os adequados a problemas de priorização de ações de intervenção, entre outros.

Os métodos ELECTRE foram desenvolvidos para, entre outros, lidar com uma limitação do SAW e TOPSIS, designadamente o facto de um mau valor num critério (possivelmente inaceitável pelo decisor) poder ser compensado por melhores valores em outros critérios. Os métodos da família ELECTRE funcionam de forma a evitar estes mecanismos compensatórios (daí serem designados métodos não-compensatórios). Além 
disso, os métodos ELECTRE acomodam de forma natural a imprecisão e incerteza dos processos de decisão, no sentido em que um decisor é geralmente insensível a pequenas diferenças nos valores dos critérios. Por último, nos métodos ELECTRE os pesos dos atributos não dependem da natureza das escalas, refletindo apenas a importância relativa entre os diferentes critérios. O ELECTRE I (e suas variantes, Is e Iv) abordam o problema da seleção da melhor alternativa de entre todas elas. $\mathrm{O}$ ELECTRE TRI aborda o problema da classificação, no sentido em que se pretende colocar as várias alternativas em classes pré- definidas (pelo AD), de acordo com o tipo de afetação, otimista ou pessimista (também definida pelo AD).

$\mathrm{O} A D$ pode interagir com $\mathrm{O} S \mathrm{SD}$ a qualquer altura do processo de decisão. Numa primeira fase, este pode selecionar ou filtrar edifícios na base de dados, focando-se naqueles cujas caraterísticas se enquadram nas suas preferências. Os parâmetros técnicos requeridos pelos diversos métodos, tais como pesos ou, nos métodos ELECTRE, limiares de indiferença, preferência ou veto, são definidos livremente pelo utilizador, o qual tem por conseguinte total controle sobre os critérios e factores de análise. O SAD permite também ao decisor efetuar uma análise de sensibilidade à variação de parâmetros, verificando na prática a influência dos limiares (de indiferença/preferência/veto) nos resultados. Esta possibilidade torna os métodos ELECTRE interessantes para a análise do tipo de problemas que aqui se pretende estudar (regeneração urbana), uma vez que permitem capturar a transição gradual do AD de um estado de indiferença para um preferência. Além disso a introdução de limiares (figura 5) permite lidar com as diferentes incertezas inerentes ao problema (p.ex. imprecisões nos dados originais) de uma forma tecnicamente justificada.

Também interessante é o facto de o ELECTRE TRI permitir uma comparação das alternativas, não entre si mas em relação a referências estáveis - as alternativas de referência - e ainda, estas alternativas de referência poderem ser totalmente parametrizadas pelo AD. Ou seja, é o AD que "cria" as alternativas de referência definindo para cada uma delas o seu desempenho em cada um dos atributos.

Por tudo o que se referiu, o ELECTRE TRI parece-nos ser particular- 
mente adequado na avaliação do estado de conservação dos edifícios em projetos de regeneração urbana. Este método permite definir os atributos dos perfis de referência utilizando quer "alternativas virtuais", quer "alternativas reais" (i.e. edifícios existentes), sendo que os últimos, pelas suas características e de acordo com o seu atual estado de conservação (EC), são para os $\mathrm{AD}$ representativos dos limites (inferior ou superior) de uma determinada classe, podendo-se assim afectar em função dessa definição os edifícios a classes do EC.

O Web-SADE, ao incorporar um SIG, permite ainda que os resultados da análise sejam visualizados em mapas virtuais (Figura 6) onde os edifícios sob avaliação aparecem devidamente localizados com uma codificação de cores que representa a respectiva classificação. Esta funcionalidade parece-nos muito útil, uma vez que permite às entidades gestoras, no desenvolvimento das suas ações de planeamento, gerar mapas com o EC dos edifícios que permitem uma visão mais ampla das reais necessidades e que serão muito úteis, por exemplo, na definição das prioridades de intervenção para o conjunto dos edifícios em análise.

\section{APlicaÇÃo A UM CASO REAL: REgENERAÇão da BAIXA de COIMBRA}

Coimbra é uma cidade histórica portuguesa, a $4 .^{\mathrm{a}}$ mais populosa de Portugal continental, se excluirmos as cidades de média dimensão que constituem as áreas metropolitanas de Lisboa e Porto. Com 96 mil habitantes (INE, Censos 2011), situa-se no litoral centro do país e possui, há mais de 700 anos, a primeira universidade portuguesa e uma das mais antigas da Europa. Nas últimas duas décadas, consequência da descentralização da habitação e das atividades económicas e comerciais (nomeadamente grandes superfícies), o seu centro histórico - "alta universitária" e "baixa da cidade" (englobando as antigas freguesias de São Bartolomeu, Almedina, Santa Cruz e Sé Nova), que até ao início do XX constituíam o núcleo urbano da cidade - tem vindo a sofrer uma perda populacional significativa, não só para as novas áreas de expansão urbana da cidade, mas também para a zona periurbana e concelhos limítrofes.

A "Baixa" de Coimbra constitui o objecto do nosso caso de estudo. 
Abrange uma área de cerca de 15 hectares onde (à data do levantamento dos dados) existiam cerca de 750 edifícios, 1200 fracções residenciais das quais apenas cerca de 60\% estavam ocupadas por 840 famílias (quase 2000 habitantes). Existiam também cerca de 1450 fracções não- residenciais, $84 \%$ das quais ocupadas (comércio, serviços e organismos do estado).

Para contrariar este cenário, e com o objectivo de preparar um programa de regeneração urbana desta zona, em 28 de Maio de 2003, a Câmara Municipal de Coimbra (CMC) estabeleceu um protocolo com a Universidade de Coimbra/Instituto de Investigação Interdisciplinar (UC/III), cujo principal objectivo foi o desenvolvimento de um estudo Interdisciplinar - envolvendo 4 equipas de investigação da UC (cerca de 40 técnicos/investigadores) - Patologias (Departamento de Engenharia Civil), Arquitetura (Departamento de Arquitetura), Sociologia (Faculdade de Economia) e Sistemas de Apoio à Decisão em Engenharia (Departamento de Engenharia Civil). Esta última equipa foi a responsável pelo desenvolvimento e implementação do web-SADE que aqui se apresenta e que tem com função permitir armazenar, tratar e analisar a informação recolhida pelas outras equipas. Utilizadores credenciados podem aceder ao web-SADE através de um browser de internet (http://sigurb.dec.uc.pt).

Apresenta-se aqui, a utilização e aplicação do web-SADE, a um conjunto de 70 edifícios pré-selecionados, cujo principal objetivo é obter uma classificação multidimensional do EC dos edifícios.

Os resultados que virão dessa classificação serão também essenciais para obter outros dados essenciais ao planeamento de uma eventual operação de regeneração, como por exemplo:

i O esforço de intervenção a efetuar em cada edifício, isto é, o tipo de obras necessárias para colocar o edifício com o nível de qualidade estipulado para a operação de reabilitação;

ii O montante necessário para executar a reabilitação de cada um dos edifícios;

iii O retorno financeiro na sequência da intervenção de reabilitação no edifício, que dependerá do potencial de valorização do edifício em função do seu estado inicial e do tipo de intervenção de reabilitação;

iv As reais necessidades e custos de realojamento dos residentes 
para cada um dos edifícios.

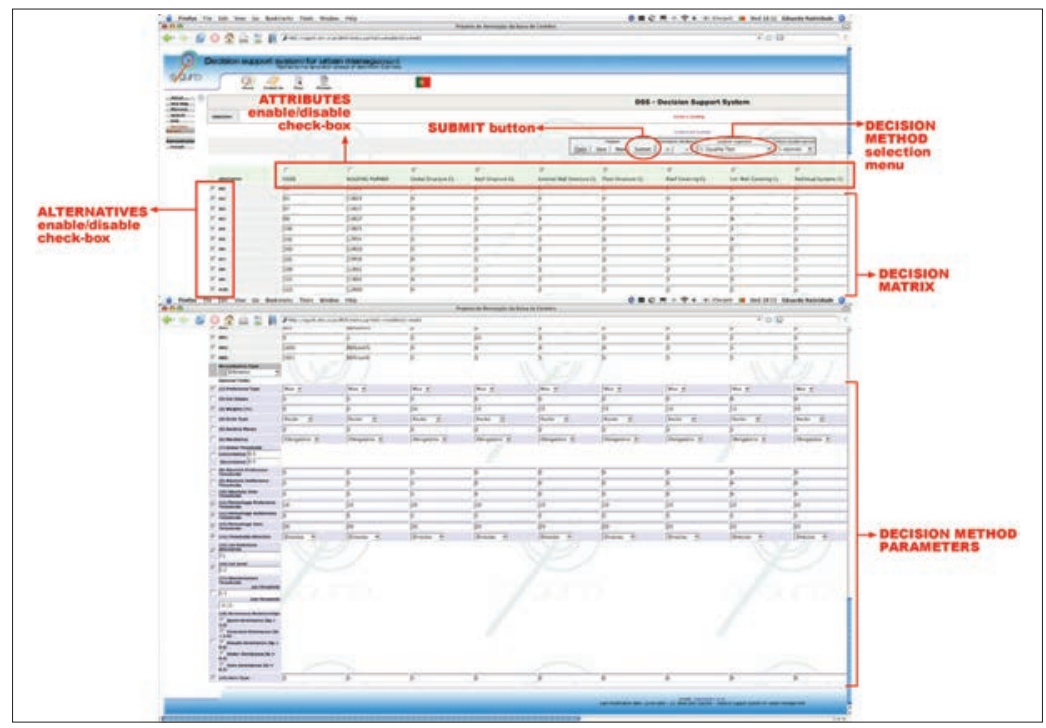

Figura 5 - Matriz de decisão e introdução de pesos e limiares para cada critério (aplicação ao caso real de 70 edifícios na Baixa de Coimbra)

O web-SADE providencia um interface com a matriz de decisão preenchida com os atributos dos edifícios selecionados para análise e subsequente classificação (aqui os edifícios são as alternativas). Os valores de cada atributo de cada edifício são recuperados automaticamente da base de dados relacional. Na parte de baixo da janela encontra-se o conjunto de parâmetros que assistem ao método de apoio à decisão a ser usado e que pode ser manipulado pelo utilizador.

Ao ser selecionado o ELECTRE TRI, o sistema desencadeia a edição dos parâmetros necessários (pesos, limiares) (figura 5). Alguns destes parâmetros podem ser introduzidos tanto em valor absoluto como em percentagem. Os atributos considerados neste estudo multicritério foram os níveis de conservação de: telhados (estrutura e cobertura), paredes exteriores (estrutura e cobertura), chão, redes de água/esgotos/eletricidade/ gás/telecomunicações.

Ao premir o botão "Submeter" na janela da matriz de decisão e parâmetros, os valores dos atributos das alternativas são enviados para os 
métodos de apoio à decisão e os módulos de resolução geram os resultados. O ELECTRE TRI devolve um output em que as alternativas são classificadas em classes com graduação por cores. A classificação resultante pode ser de imediato representada na componente SIG do web-SADE na correspondente graduação (figura 6) (mais escuro corresponde a mais degradado). Neste caso foram consideradas seis classes, de "novo" a "em ruínas”. A figura evidencia um estado global de alguma degradação, justificando a necessidade de ações de regeneração.

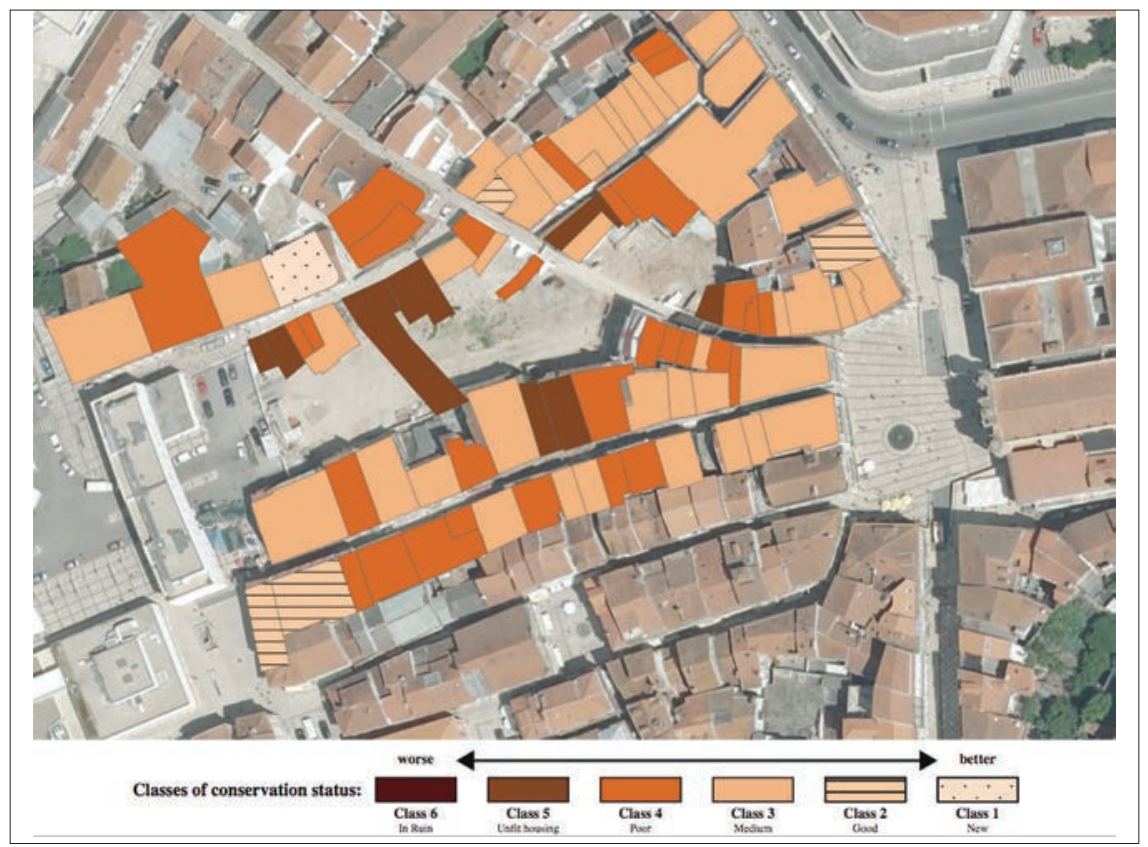

Figura 6 - Afetação dos edifícios a classes de NQC (aplicação ao caso real de edifícios na Baixa de Coimbra)

Como referido acima, o utilizador pode interagir com o SAD em qualquer fase do processo de decisão. Pode por exemplo ativar ou desativar atributos por via da checkbox respetiva na janela de edição do interface web, alterar parâmetros (pesos e/ou limiares) e obter novos resultados. Outputs sucessivos podem ser obtidos e salvos no sistema, permitindo ao utilizador comparar posteriormente resultados para o mesmo método (com parâmetros diferentes), ou entre os vários métodos. Isto é extremamente útil para a supracitada análise de sensibilidade e facilita o primeiro contacto do utilizador com o interface e com os métodos, reforçando assim 
o seu conhecimento e intuição sobre o processo de decisão, o qual estará sempre sobre o seu controle.

\section{ConcluSÃo}

A segunda metade do Séc. XX assistiu a um rápido crescimento das zonas urbanas na maioria dos países desenvolvidos. Este crescimento, acoplado à degradação dos centros urbanos, tem importantes consequências socioeconómicas e ambientais.

Para mitigar estas tendências, muitos países estão a tentar renovar e/ ou regenerar as suas cidades, em especial os centros históricos destas. No entanto, tais esforços são extremamente complexos e dispendiosos, o que justifica o desenvolvimento e uso da mais recente tecnologia em métodos de apoio à decisão para auxiliar os decisores.

A análise do nível de EC dos edifícios é um passo crucial no estudo deste tipo de problemas. Esta avaliação envolve a determinação sistémica dos fatores que influenciam o EC de um edifício, baseados em princípios claros e usando critérios bem definidos. As múltiplas dimensões do problema evidenciam a necessidade de se aplicar métodos multicritério cientificamente comprovados. Tal aplicação depende da qualidade da informação recolhida mas também das possibilidades de escolha oferecidas ao utilizador. O sistema web-SADE apresentado neste trabalho tem como objetivo oferecer ao AD uma ferramenta flexível e de fácil utilização, baseada em métodos multicritério formais, para o assistir na recolha, armazenamento e estruturação da informação relevante, na análise de histórico e estatística e, sobretudo, para lhe providenciar ajuda à tomada de decisão, permitindo uma comparação racional entre as várias alternativas ou cenários da intervenção de regeneração urbana.

Por último, o presente trabalho apresenta como aplicação do web-SADE um estudo de caso sobre a Baixa da cidade de Coimbra, Portugal. Neste estudo o método multicritério usado, ELECTRE TRI, revelou ser especialmente adequado à classificação e seleção de edifícios. Na verdade, permite definir edifícios de referência, no que respeita ao EC, e classificar todos os outros por comparação com os primeiros. 


\section{AGRADECIMENTOS}

Este trabalho foi parcialmente financiado pela Fundação Portuguesa para a Ciência e Tecnologia (FCT) sob a Ref "PEst-OE/ EEI/UI308/2014", pelo projeto Energy and Mobility for Sustainable Regions (EMSURE) - Ref ${ }^{a}$ CENTRO-07-0224-FEDER-002004, e enquadra-se na iniciativa Energy for Sustainability da Universidade de Coimbra.

\section{BIBLIOGRAFIA}

ANDERSEN, H.T. \& VAN KEMPEN, R. (2003) - "New trends in urban policies in Europe: evidence from the Netherlands and Denmark." Cities, Vol. 20, $\mathrm{N}^{\circ}$ 2, 77-86.

BAI et al. (2010) - "Urban policy and governance in a global environment: complex systems, scale mismatches and public participation," Current Opinion in Environmental Sustainability, Vol. 2, $\mathrm{N}^{\mathrm{o}} 3,129-135$.

BREDA-VÁZQUEZ, I. (2009) - "Partnership Diversity and Governance Culture: Evidence from Urban Regeneration Policies in Portugal." Urban Studies, Vol. 46, No 10, 2213- 2238.

CAPUTO, S. et al. (2012) - "Testing energy efficiency in urban regeneration." Proceedings of the ICE - Engineering Sustainability, Vol. 165, No 1, 69-80.

CHURCHMAN CW \& ACKOFF RL (1954) - "An appropriate measure of value." Journal of Operations Research Society of America, Vol. 2, $N^{\circ}$ 2, 172-187.

COPENHAGEN ECONOMICS (2012) - "Renovate Europe - Multiple Benefits of Investing in Energy Efficient Renovations - Impact on Public Finances."

DIÁRIO DA REPÚBLICA (2004) Decreto-lei no 104/2004. Diário da República - I Série $\mathrm{A}, \mathrm{n}^{\circ}$ 107, de 7 de maio de 2004, 2920-2929. Imprensa Nacional Casa da Moeda, Lisboa, Portugal.

DIÁRIO DA REPÚBliCA (2005) Decreto-lei no 52/2005 - Grandes Opções do Plano 2005- 2009. Diário da República - I Série A, no 167, de 31 de agosto de 2005, 5186-5284. Imprensa Nacional Casa da Moeda, Lisboa, Portugal.

DIÁRIO DA REPÚBLICA (2007) Resolução do Conselho de Ministros nº 109/2007 - Estratégia Nacional de Desenvolvimento Sustentátvel. Diário da República - I Série, $\mathrm{n}^{\circ}$ 159, de 20 de agosto de 2007, 5404-5478. Imprensa Nacional Casa da Moeda, Lisboa, Portugal.

DING, G.K. (2008) - "Sustainable construction - The role of environmental assessment tools.” Journal of Environmental Management, Vol. 86, 451-464. 
ENERDATA \& ADEME - AGENCE DE L'ENVIRONNEMENT ET DE LA MAÎTRISE DE L'ENERGIE (2012) - "Energy Efficiency Trends in Buildings in the EU." Odyssee Database, 67.

EUROCONSTRUCT (2009) - "Country Report - 68th Euroconstruct Conference," EUROCONSTRUCT, Zurich.

KAZMIERCZAK, A.E. et al. (2009) - "Regeneration of large urban areas: assessment methods." Proceedings of the ICE - Municipal Engineer, Vol. 62, $\mathrm{N}^{\circ}$ 2, $117-124$.

LANE, M.B. (2005) - "Public Participation in Planning: an intellectual history." Australian Geographer, Vol. 36, N 3, 283-299.

MALCZEWSKI, J (2004) - "GIS-based land-use suitability analysis: a critical overview." Progress in Planning, Vol. 62, $\mathrm{N}^{\circ}$ 1, 3-65.

MASSAM BH (1988) - "Multi-criteria Decision Making (MCDM) Techniques in Planning." Progress in Planning, Vol. 30, $\mathrm{N}^{\circ} 1,1-84$.

NATIVIDADE-JESUS, E. et al. (2007) - "A multicriteria decision support system for housing evaluation.” Decision Support Systems, Vol. 43, 779-790.

NATIVIDADE-JESUS, E. et al. (2013) -"Housing Evaluation in Urban Renovation using web - based Multicriteria SDSS.”, Municipal Engineer, Vol, 166, N 3, $194-207$.

ORUETA, F.D. (2007) - "Madrid: Urban regeneration projects and social mobilization." Cities, Vol. 24, No 3, 183-193.

PRICE, L. et al. (2006) - "Sectoral Trends in Global Energy Use and GHG Emissions." Lawrence Berkeley National Laboratory.

ROY, B (1991) - "The outranking approach and the foundations of ELECTRE methods." Theory and Decision, Vol. 31, 49-73.

SCHÄRLIG, A (1996) - Pratiquer Electre et Prométhée - Un Complément à Décider sur Plusieurs Critères. Collection Diriger L'Entreprise, Vol. 11, Presses Polytechniques et Universitaires Romandes, Lausana, Suíça.

UNFCCC (1997) - Kyoto Protocol to the United Nations Framework Convention on Climate Change. Kyoto, Japan. Disponível online no endereço url: http:// unfccc.int/resource/docs/convkp/kpeng.html (acedido em 30 março, 2014).

UNIÃO EUROPEIA (2003) - Diretiva 2002/91/CE do Parlamento Europeu e do Conselho, de 16 de dezembro de 2002, relativa ao desempenho energético dos edifícios. Disponível online no endereço url: http://eur- lex.europa. eu/LexUriServ/LexUriServ.do?uri=OJ:L:2003:001:0065:0065:PT:PDF (acedido em 30 março, 2014).

UNIÃO EUROPEIA (2003) - Diretiva 2010/31/CE do Parlamento Europeu e do Conselho, de 19 de Maio, relativa ao desempenho energético dos edifícios. Disponível online no endereço url: (acedido em 30 março, 2014). http:// 
eur- lex.europa.eu/LexUriServ/LexUriServ.do?uri=OJ:L:2010:153:0013:003 5:PT:PDF

VASCONCELOS, L. et al. (2009) - Governância e participação na gestão territorial. Lisboa: Série Política de Cidades, 2009.

YOON, KP \& HWANG, CL (1995) - Multiple Attribute Decision-Making: An Introduction. Sage University papers, Series on Quantitative Applications in the Social Science, 7- 104, 73 p.

ZAVADSKAS, E. et al. (2008) - "The selection of effective retrofit scenarios for panel houses in urban neighbourhoods based on expected energy savings and increase in market value: The Vilnius case." Energy Buildings, Vol. 40, $\mathrm{N}^{\mathrm{o}} 4,573-587$. 
Série Documentos

Imprensa da Universidade de Coimbra

Coimbra University Press

2015

- U M

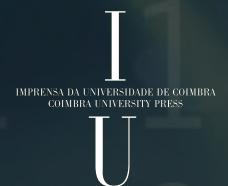

